BRAVZULIAN JOURNAL

OF MEDICAL AND BIOLOGICAL RESH.ARCH

www.bjournal.com.br
ISSN 0100-879X

Volume 44 (6) 497-605 June 2011

BIOMEDICAL SCIENCES

AND

CLINICAL INVESTIGATION

Braz J Med Biol Res, June 2011, Volume 44(6) 583-591

doi: 10.1590/S0100-879X2011007500053

Morphometric analysis of the phrenic nerve in male and female Wistar-Kyoto (WKY) and spontaneously hypertensive rats (SHR)

A.R. Rodrigues, R.S. Ferreira, H.C. Salgado and V.P.S. Fazan

The Brazilian Journal of Medical and Biological Research is partially financed by



Institutional Sponsors

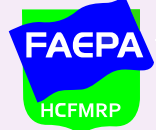

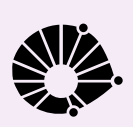

UNICAMP
Ф) SHIMADZU

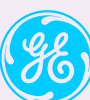

GE Healthcare
Hotsite of proteomics metabolomics developped by:






\title{
Morphometric analysis of the phrenic nerve in male and female Wistar-Kyoto (WKY) and spontaneously hypertensive rats (SHR)
}

\author{
A.R. Rodrigues ${ }^{1}$, R.S. Ferreira1 ${ }^{1}$, H.C. Salgado ${ }^{2}$ and V.P.S. Fazan ${ }^{1}$ \\ 1Departamento de Neurociências e Ciências do Comportamento, ${ }^{2}$ Departamento de Fisiologia, \\ Faculdade de Medicina de Ribeirão Preto, Universidade de São Paulo, Ribeirão Preto, SP, Brasil
}

\begin{abstract}
Ventilatory differences between rat strains and genders have been described but the morphology of the phrenic nerve has not been investigated in spontaneously hypertensive (SHR) and normotensive Wistar-Kyoto (WKY) rats. A descriptive and morphometric study of the phrenic nerves of male $(N=8)$ and female $(N=9)$ SHR, and male $(N=5)$ and female $(N=6)$ WKY is presented. After arterial pressure and heart rate recordings, the phrenic nerves of 20 -week-old animals were prepared for epoxy resin embedding and light microscopy. Morphometric analysis was performed with the aid of computer software that took into consideration the fascicle area and diameter, as well as myelinated fiber profile and Schwann cell nucleus number per area. Phrenic nerves were generally larger in males than in females of both strains but larger in WKY compared to SHR for both genders. Myelinated fiber numbers (male SHR $=228 \pm 13$; female SHR $=258 \pm 4$; male WKY $=382 \pm 23$; female WKY $=$ $442 \pm 11$ for proximal right segments) and density ( $/ \mathrm{mm}^{2}$; male SHR = $7048 \pm 537$; female SHR = $10355 \pm 359$; male WKY = $9454 \pm 1437$; female WKY = $14361 \pm 1448$ for proximal right segments) were significantly larger in females of both groups and remarkably larger in WKY than SHR for both genders. Strain and gender differences in phrenic nerve myelinated fiber number are described for the first time in this experimental model of hypertension, indicating the need for thorough functional studies of this nerve in male and female SHR.
\end{abstract}

Key words: Phrenic nerve; Sexual dimorphism; Morphometry; Spontaneously hypertensive rats; Wistar-Kyoto rats; Asymmetry

\section{Introduction}

Animal studies have provided evidence for genetic transmission of ventilatory traits. Differences among inbred rat strains (1), as well as differences between inbred and outbred rat strains (2), have been documented during eupnea, hypoxia, hypercapnia, and exercise $(2,3)$. Some of these differences are associated with genotypic differences localized on specific chromosomes (3). Ventilatory differences between male and female rats during eupnea, hyperoxia, hypoxia, or hyperoxia hypercapnia have been described $(2,4)$ for the same rat strain. Moreover, gender differences in ventilatory chemosensitivity and particularly in the ventilatory response to hypoxia have been examined in a number of studies $(2,4)$.

The phrenic nerve of rats is the main nerve involved in respiratory control because it is the only source of innervation for the diaphragm (5). Histological examination of the diaphragm has shown gender differences in fiber composition and morphometry (6), but gender differences in phrenic nerve morphology and morphometry have not been examined. Studies that investigated the interaction between hypertension, gender and respiratory control have mainly focused on sleep apnea syndrome (7), and male gender and hypertension have been suggested to be risks for human sleep apnea (8). Animal models have been useful to investigate the relationship between hypertension and abnormal control of breathing (9). Despite recent literature reviews focusing on the relationship between breathing and hypertension $(10,11)$, a possible relationship between hypertension and abnormal ventilatory control was never examined before in spontaneously hypertensive rats (SHR), an outstanding hypertensive model. Moreover, the literature lacks a thorough description of phrenic nerve morphology and morphometry in this experimental model and in the normotensive controls, the Wistar-Kyoto (WKY) rats.

Correspondence: V.P.S. Fazan, Departamento de Neurociências e Ciências do Comportamento, FMRP, USP, 14049-900 Ribeirão Preto, SP, Brasil. Fax: +55-16-3633-0017. E-mail: vpsfazan@yahoo.com.br or vpsfazan@gmail.com

Received September 21, 2010. Accepted April 15, 2011. Available online May 2, 2011. Published June 13, 2011. 
To test the hypothesis that the phrenic nerve might be responsible, at least in part, for the gender differences in ventilatory control described previously $(2,4)$ and also to investigate the possible influence of the hypertensive state on phrenic nerve morphology, the objective of the present study was to describe and to compare morphometric parameters of the phrenic nerves in male and female SHR and WKY, with a lateral and longitudinal symmetry study of this nerve. Gender differences were investigated in each animal strain and the influence of hypertension on phrenic nerve morphometry was assessed by modern computer techniques.

\section{Material and Methods}

Experiments were performed on SHR and WKY, born and raised in the animal care facility of the Department of Neuroscience and Behavioral Neuroscience, School of Medicine of Ribeirão Preto, under controlled room temperature $\left(21-23^{\circ} \mathrm{C}\right)$ and air humidity $(40-70 \%)$ and dark/light cycle of $12 \mathrm{~h}$. The animals were housed in plastic cages (3-4 animals to a cage) with free access to tap water and rat chow. All experimental procedures adhered to the Guide for the Care and Use of Laboratory Animals prepared by the United States National Academy of Sciences and published by the National Institutes of Health (Copyright ${ }^{\complement} 1996$ by the National Academy of Sciences), and were approved by the Institutional Ethics Committee for Animal Research (CETEA - Comitê de Ética em Experimentação Animal, protocol \#062/2007). All efforts were made to minimize the number of animals used.

Male $(\mathrm{N}=8)$ and female $(\mathrm{N}=9) \mathrm{SHR}$, and male $(\mathrm{N}=5)$ and female $(\mathrm{N}=6) \mathrm{WKY}, 20$ weeks old, were anesthetized with sodium thiopental (Thionembutal, $40 \mathrm{mg} \mathrm{kg}$, ip) and a catheter was inserted into the femoral artery for measurement of arterial pressure (AP). Recordings of the systolic (SAP), diastolic (DAP) and mean arterial pressure (MAP) and heart rate (HR) were performed as described elsewhere (12). After the recordings, the rats were perfused through the left ventricle with $50 \mathrm{mM}$ phosphate-buffered saline (PBS), $\mathrm{pH} 7.4$, followed by $2.5 \%$ glutaraldehyde solution in $0.1 \mathrm{M}$ cacodylate buffer, $\mathrm{pH}$ 7.2. Both right and left phrenic nerves were carefully dissected between the clavicle and diaphragm level, removed in one piece, affixed to a strip of filter paper and placed in the fixative solution for an additional $12 \mathrm{~h}$. Next, they were washed in cacodylate buffer, $\mathrm{pH} 7.2$, and a proximal segment (close to the clavicle) and a distal segment (before entering the diaphragm) of approximately $3 \mathrm{~mm}$ each were separated and processed for epoxy resin embedding (EMbed-812 ${ }^{\circledR}$, Electron Microscopy Sciences, USA) as described previously $(13,14)$. The remaining nerve fragment was kept in $0.1 \mathrm{M}$ cacodylate buffer, $\mathrm{pH} 7.2$, at $4^{\circ} \mathrm{C}$ for further study. The methods for histological preparation of the nerves were described previously $(15,16)$. Ten consecutive semithin (0.2-0.3 $\mu \mathrm{m}$ thick) transverse sections of the fascicle segments were stained with $1 \%$ Toluidine blue and examined with the aid of an Axiophot II photomicroscope (Carl Zeiss, Germany). The images were sent via a digital camera (TK-1270, JVC, Victor Company of Japan Ltd., Japan) to an IBM/PC where they were digitized. For the study of myelinated fibers the endoneural space was observed under an optical set equipped with an oil immersion lens (10X), optovar (1.6X), camera (0.5X), and a computerized magnification of $8 X$ provided images with good resolution for morphometry.

The endoneural space was fully scanned without overlap of the microscopic fields using an automated motorized stage (Carl Zeiss). Scannings generated 6 to 12 microscopic fields of $640 \times 470$ pixels, which were used to count and automatically measure the myelinated fibers and respective axons. Fibers at the upper and left edges of the microscopic fields were counted, whereas those at the lower and right edges were not counted ("forbidden line"), in order to avoid counting the same fiber twice. All myelinated fibers (defined by the axon and its respective myelin sheath, excluding the Schwann cell nucleus when present) in the endoneural space were counted. The program was able to identify, and delete automatically, fibers set at the edge of the microscopic field under study. Morphometric parameters of the fascicles and the myelinated fibers of the phrenic nerve segments were obtained as described previously (17). Briefly, the total number of myelinated fibers and the profile of total number of Schwann cell nuclei present in each fascicle were counted and estimated per area using the 2-D morphometry approach. The area and lesser diameter of each fascicle (excluding the perineurium) and of each myelinated fiber and respective axon (fiber without the myelin sheath envelopment) were measured with the image analysis software (KS 400, Kontron 2.0, Eching Bei München, Germany). The lesser diameter is the one that better represents the diameter of a non-circular fascicle and fiber $(16,18)$. The percentage of the total cross-sectional area of the endoneural space occupied by the myelinated fibers was calculated, and hereafter referred to as percentage of occupancy of the myelinated fibers $(14,16)$. The densities of the myelinated fibers and the profile of Schwann cell nuclei were estimated per area. For the myelinated fibers, both axonal diameter and total fiber diameter were automatically measured. The ratio between the two diameters, i.e., the $G$ ratio (which indicates the degree of myelination) (19), was obtained. The myelin sheath area was calculated for each myelinated fiber measured. Histograms of population distribution of myelinated fibers and axons, divided into class intervals increasing by $1.0 \mu \mathrm{m}$, were constructed. Histograms of the $\mathrm{G}$ ratio (axon diameter/fiber diameter) distribution divided into class intervals increasing by 0.1 were also constructed.

Physiological data were tested for normal distribution by the Kolmogorov-Smirnov normality test, followed by the Levene test for variance equivalence. If data presented a 
normal distribution and equivalent variance, comparisons were made between groups by the unpaired Student $t$ test. Otherwise, the non-parametric Mann-Whitney test was used. Morphometric data were also tested for normal distribution by the Kolmogorov-Smirnov normality test, followed by the Levene test for variance equivalence. If data presented a normal distribution and equivalent variance, comparisons were made between proximal and distal segments in the same group by the paired Student $t$-test. Otherwise, comparisons were made by the non-parametric Wilcoxon test for paired samples. For comparisons between right and left segments in the same group and between genders, normally distributed data were tested using the unpaired Student $t$-test. Alternatively, comparisons were made by the non-parametric Mann-Whitney test. Comparisons between histograms were made by one-way analysis of variance (ANOVA) on ranks, provided that the distributions did not pass the normality test. Differences were considered to be significant if $P<0.05$. Data are reported as means \pm SEM.

\section{Results}

\section{Blood pressure and heart rate data}

Male and female SHR showed HR of $381 \pm 10$ and $320 \pm 10 \mathrm{bpm}$, respectively, with a significant difference between genders. For WKY, male and female HR was $363 \pm 14$ and $363 \pm 20$, respectively, with no differences between genders. HR differences were observed between strains for both genders.

The blood pressure data of male and female SHR and WKY are shown in Figure 1. Male SHR showed remarkably higher SAP values than female SHR. No significant differences were observed in MAP or DAP between SHR genders. No differences in SAP, MAP or DAP were observed between genders in WKY animals.

\section{Morphological data}

The phrenic nerve consisted of a single fascicle in most segments studied for both SHR genders (Figure 2), in agreement with previous descriptions for other animal strains $(17,20)$. No clear morphological differences were observed between segments, sides or genders. The phrenic nerves of WKY animals (Figure 3 ) were morphologically similar to SHR nerves, also with no evident differences between segments, sides or genders. Nevertheless, observation of the cross-sections at the same magnification (Figure 3) clearly showed that WKY nerves were larger than SHR nerves for all segments studied.

\section{Fascicular morphometry - comparison between genders}

Morphometric data corresponding to fascicular area and diameter, estimated number and density of myelinated fibers, estimated number and density of Schwann cell nuclei, myelinated fiber percentage of occupancy (percentage of the total cross-sectional area of the endoneural space occupied by the myelinated fibers - axon and respective myelin sheath), and capillary percentage of occupancy (percentage of the luminal cross-sectional area of all capillaries present in the endoneural space) are shown in Table 1 (SHR) and Table 2 (WKY). Phrenic nerve fascicles were generally larger in male than in female SHR. Female SHR showed significantly larger estimated number and density of myelinated fibers than male SHR.

Both SHR genders showed a smaller fiber percentage of occupancy in proximal segments of both sides, with a significant difference between the left proximal and distal segments. No differences in percent fiber occupancy or percent capillary occupancy were observed between genders in SHR.

For the WKY phrenic nerves, a trend towards a smaller size (area and diameter) was observed from proximal to distal segments on both sides and in both genders, with no significant differences. As observed for SHR, WKY phrenic nerves were generally larger in males than in females with significant differences observed on the left side. Regarding the estimated number of myelinated fibers and respective densities, female WKY showed significantly higher values than male WKY in all segments studied. No differences



Figure 1. Average systolic (SAP), mean (MAP) and diastolic (DAP) arterial pressure of male $(\mathrm{N}=8)$ and female $(\mathrm{N}=9)$ spontaneously hypertensive rats $(\mathrm{SHR})$. ${ }^{*} \mathrm{P}<0.05$ compared to male SHR; ${ }^{\mathrm{P}}<0.05$ compared to female SHR; ${ }^{+} \mathrm{P}<0.05$ compared to male Wistar-Kyoto (WKY); ${ }^{\circ} \mathrm{P}<0.05$ compared to female WKY (oneway ANOVA). 

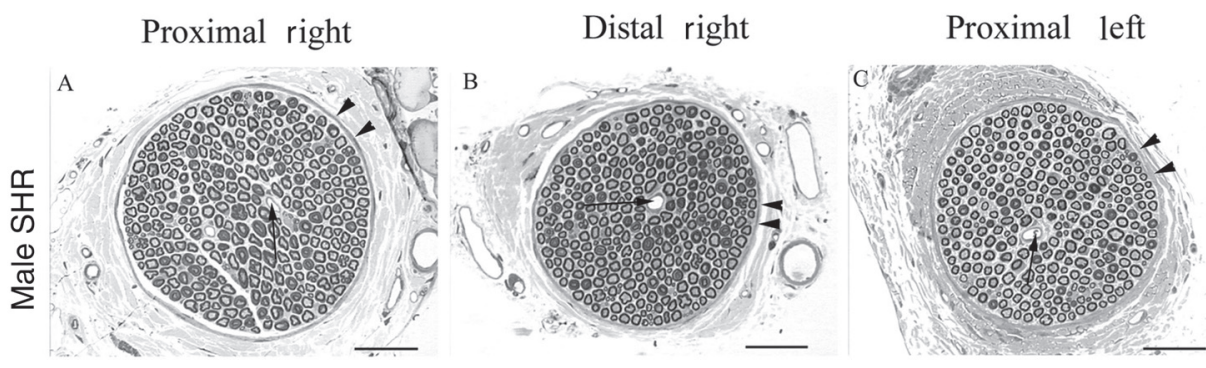

Distal left


Figure 2. Transverse sections of the right $(A, B, E$, and $F)$ and left $(C, D, G$, and $H)$ phrenic nerves of male $(A, B, C$, and $D)$ and female $(E, F, G$, and $H)$ spontaneously hypertensive rats (SHR). Proximal $(A, B, E$, and $G)$ and distal $(B, D, F$, and $H)$ segments are shown. Note that the phrenic nerves of SHR are monofasciculated and each fascicle is enveloped by a well-defined perineurium (arrowheads). The endoneural space contains hundreds of myelinated and unmyelinated fibers, and one or two blood vessels (arrows) accompany the longitudinal axis of the fascicles. No morphological differences were observed between male and female SHR. Toluidine blue staining; bars $=50 \mu \mathrm{m}$.

Male WKY

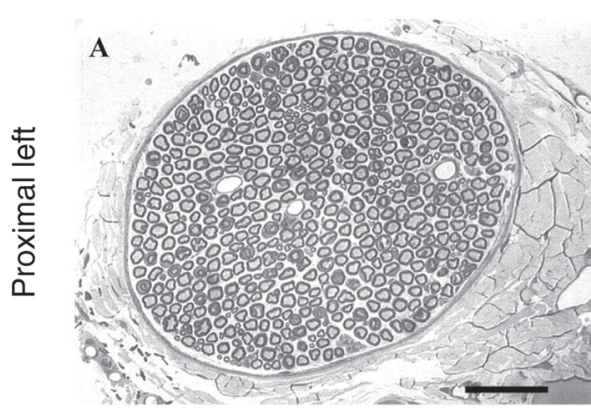

\section{Female WKY}

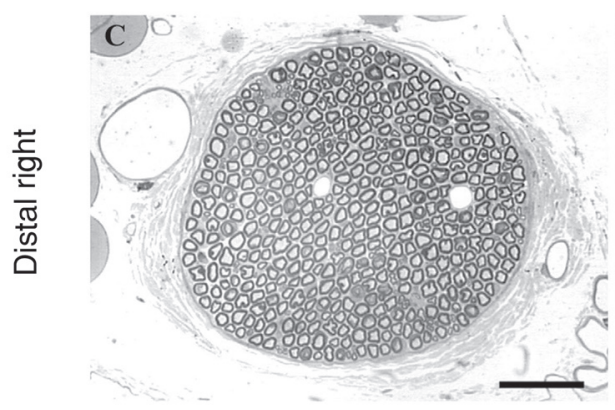

Male SHR

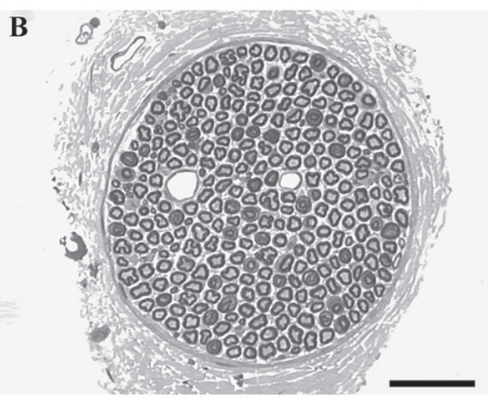

Female SHR

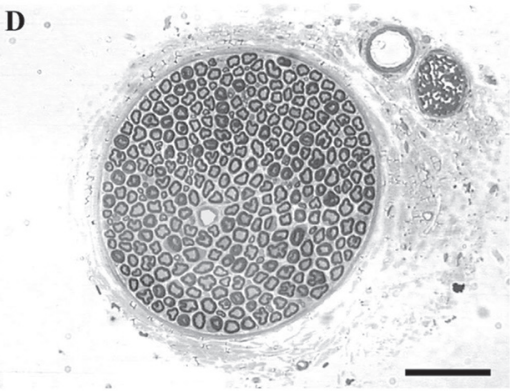

Figure 3. Representative transverse sections of the phrenic nerves of male and female WistarKyoto (WKY; A, C) rats and male and female spontaneously hypertensive rats (SHR; B, D). Note that WKY nerves are generally larger than SHR nerves in both genders. There is also a trend towards a smaller nerve size in females compared to males of the same strain. Toluidine blue staining; bars $=50 \mu \mathrm{m}$ 
were observed between segments, sides or genders for the estimated profiles of Schwann cell nucleus number and density in WKY phrenic nerves.

Fiber percentage of occupancy was significantly smaller in male WKY compared to females in all segments studied. The capillary percentage of occupancy did not differ between sides, segments or genders in WKY phrenic nerves.

\section{Fascicular morphometry - comparison between SHR} and WKY

Overall, the phrenic nerves of WKY were significantly larger (area and diameter) than those of SHR for all segments studied, with no specificity for a particular side or segment. This observation was also true for the estimated number and density of myelinated fibers, with the estimated

Table 1. Morphometric data of the phrenic nerve fascicle of 20-week-old male and female spontaneously hypertensive rats (SHR).

\begin{tabular}{|c|c|c|c|c|c|c|c|c|}
\hline \multirow{3}{*}{$\begin{array}{l}\text { Fascicle } \\
\text { parameter }\end{array}$} & \multicolumn{4}{|c|}{ Male SHR $(\mathrm{N}=8)$} & \multicolumn{4}{|c|}{ Female SHR $(\mathrm{N}=9)$} \\
\hline & \multicolumn{2}{|c|}{ Proximal } & \multicolumn{2}{|c|}{ Distal } & \multicolumn{2}{|c|}{ Proximal } & \multicolumn{2}{|c|}{ Distal } \\
\hline & Right & Left & Right & Left & Right & Left & Right & Left \\
\hline Area $\left(\mu \mathrm{m}^{2}\right)$ & $32805 \pm 1467^{*}$ & $27780 \pm 1300^{\#}$ & $28509 \pm 816^{*}$ & $23166 \pm 1132^{\#+}$ & $25219 \pm 1119$ & $25292 \pm 1748$ & $23930 \pm 1155$ & $20768 \pm 1151$ \\
\hline Diameter $(\mu \mathrm{m})$ & $191 \pm 6^{*}$ & $178 \pm 6$ & $183 \pm 4^{*}$ & $161 \pm 4^{* \#+}$ & $165 \pm 6$ & $163 \pm 6$ & $161 \pm 5$ & $143 \pm 4^{\#+}$ \\
\hline $\mathrm{MF}(\mathrm{N})$ & $228 \pm 13^{*}$ & $220 \pm 6^{*}$ & $229 \pm 10^{*}$ & $228 \pm 5^{*}$ & $258 \pm 4$ & $241 \pm 7$ & $262 \pm 6$ & $240 \pm 8$ \\
\hline $\begin{array}{l}\text { MF density } \\
\left(\mathrm{N} / \mathrm{mm}^{2}\right)\end{array}$ & $7048 \pm 537^{*}$ & $8070 \pm 516^{*}$ & $8086 \pm 471^{*}$ & $10036 \pm 648^{*}$ & $10355 \pm 359$ & $9818 \pm 649$ & $11101 \pm 511$ & $11805 \pm 679$ \\
\hline $\begin{array}{l}\text { Number of SC } \\
\text { nuclei }(N)\end{array}$ & $6 \pm 1$ & $5 \pm 1^{*}$ & $6 \pm 1$ & $6 \pm 1$ & $4 \pm 1$ & $3 \pm 1$ & $4 \pm 1$ & $4 \pm 1$ \\
\hline $\begin{array}{l}\text { Density of SC } \\
\text { nuclei }\left(\mathrm{N} / \mathrm{mm}^{2}\right)\end{array}$ & $176 \pm 29$ & $163 \pm 21$ & $196 \pm 27$ & $291 \pm 75$ & $153 \pm 36$ & $138 \pm 33$ & $189 \pm 24$ & $216 \pm 42$ \\
\hline $\begin{array}{l}\text { Fiber } \\
\text { occupancy (\%) }\end{array}$ & $39 \pm 3$ & $39 \pm 1^{+}$ & $41 \pm 1$ & $45 \pm 2$ & $41 \pm 1$ & $39 \pm 1^{+}$ & $42 \pm 1$ & $43 \pm 1$ \\
\hline $\begin{array}{l}\text { Capillary } \\
\text { occupancy (\%) }\end{array}$ & $0.3 \pm 0.05$ & $0.4 \pm 0.14$ & $0.6 \pm 0.2$ & $0.14 \pm 0.05^{\#+}$ & $0.4 \pm 0.13$ & $0.4 \pm 0.1$ & $0.6 \pm 0.2$ & $0.2 \pm 0.06^{\#+}$ \\
\hline
\end{tabular}

Data are reported as means \pm SEM. MF = myelinated fiber; $S C=$ Schwann cell; $N=$ number. ${ }^{*} P<0.05$, male SHR compared to female SHR (unpaired $t$-test); \# $\mathrm{P}<0.05$, between sides (unpaired $t$-test); ${ }^{+} \mathrm{P}<0.05$, proximal compared to distal gradient on the same side (paired $t$-test).

Table 2. Morphometric data of the phrenic nerve fascicle of 20-week-old male and female Wistar-Kyoto (WKY) rats.

\begin{tabular}{|c|c|c|c|c|c|c|c|c|}
\hline \multirow{3}{*}{$\begin{array}{l}\text { Fascicle } \\
\text { parameter }\end{array}$} & \multicolumn{4}{|c|}{ Male WKY $(\mathrm{N}=5)$} & \multicolumn{4}{|c|}{ Female WKY $(\mathrm{N}=6)$} \\
\hline & \multicolumn{2}{|c|}{ Proximal } & \multicolumn{2}{|c|}{ Distal } & \multicolumn{2}{|c|}{ Proximal } & \multicolumn{2}{|c|}{ Distal } \\
\hline & Right & Left & Right & Left & Right & Left & Right & Left \\
\hline Area $\left(\mu m^{2}\right)$ & $42340 \pm 4483^{+}$ & $43902 \pm 3397^{\star+}$ & $35829 \pm 1229^{+}$ & $36238 \pm 3248^{*+}$ & $32140 \pm 2832^{+}$ & $31763 \pm 2927$ & $31613 \pm 1353^{+}$ & $27776 \pm 1680^{+}$ \\
\hline Diameter $(\mu \mathrm{m})$ & $211 \pm 11$ & $220 \pm 3^{*+}$ & $187 \pm 23$ & $190 \pm 16^{+}$ & $177 \pm 13$ & $169 \pm 12$ & $169 \pm 9$ & $163 \pm 6^{+}$ \\
\hline MF (N) & $382 \pm 23^{*+}$ & $368 \pm 9^{\star+}$ & $391 \pm 12^{*+}$ & $388 \pm 15^{\star+}$ & $442 \pm 11^{+}$ & $457 \pm 10^{+}$ & $448 \pm 12^{+}$ & $470 \pm 17^{+}$ \\
\hline $\begin{array}{l}\text { MF density } \\
\left(\mathrm{N} / \mathrm{mm}^{2}\right)\end{array}$ & $9454 \pm 1437$ & $8513 \pm 647^{*}$ & $10963 \pm 628^{*+}$ & $10872 \pm 697^{*}$ & $14361 \pm 1448^{+}$ & $15009 \pm 1408^{+}$ & $14246 \pm 408^{+}$ & $17296 \pm 1366^{+}$ \\
\hline SC nuclei (N) & $9 \pm 1^{+}$ & $10 \pm 1^{+}$ & $9 \pm 2^{+}$ & $10 \pm 2$ & $9 \pm 1^{+}$ & $7 \pm 1^{+}$ & $9 \pm 1^{+}$ & $8 \pm 1^{+}$ \\
\hline $\begin{array}{l}\text { SC nuclei density } \\
\left(\mathrm{N} / \mathrm{mm}^{2}\right)\end{array}$ & $237 \pm 41$ & $231 \pm 11^{+}$ & $264 \pm 58$ & $282 \pm 56$ & $288 \pm 30^{+}$ & $225 \pm 40$ & $286 \pm 34^{+}$ & $292 \pm 30$ \\
\hline $\begin{array}{l}\text { Fiber } \\
\text { occupancy (\%) }\end{array}$ & $41 \pm 3^{*}$ & $41 \pm 3^{*}$ & $42 \pm 3^{*}$ & $42 \pm 3^{*}$ & $50 \pm 2^{+}$ & $53 \pm 2^{+}$ & $51 \pm 2^{+}$ & $55 \pm 2^{+}$ \\
\hline $\begin{array}{l}\text { Capillary } \\
\text { occupancy (\%) }\end{array}$ & $0.2 \pm 0.08$ & $0.3 \pm 0.04$ & $0.3 \pm 0.15$ & $0.2 \pm 0.16$ & $0.6 \pm 0.16$ & $1.0 \pm 0.3^{+}$ & $0.9 \pm 0.3$ & $0.5 \pm 0.1^{+}$ \\
\hline
\end{tabular}

Data are reported as means $\pm \mathrm{SEM}$. MF = myelinated fiber; $\mathrm{SC}=\mathrm{Schwann}$ cell; $\mathrm{N}=$ number. ${ }^{*} \mathrm{P}<0.05$, male WKY compared to female WKY (unpaired $t$-test); ${ }^{+} \mathrm{P}<0.05$, compared to SHR (one-way ANOVA). No differences between segments or sides were observed. 
number of myelinated fibers of female WKY reaching values almost twice those of female SHR. The estimated profile of Schwann cell nucleus number was also significantly larger in WKY nerves in almost all segments studied and their estimated density followed this trend to a lesser extent.

Myelinated fiber occupancy was significantly larger in female WKY phrenic nerves compared to female SHR, with no differences between male WKY and SHR. No differences in capillary occupancy were observed between WKY and SHR nerves.

\section{Myelinated fiber morphometry - comparison between genders}

The area and diameter of the myelinated fibers and respective axons of the phrenic nerves of SHR and WKY were generally larger in males but few significant differences were observed between genders. Few scattered intra-group differences were observed between segments or sides and were more evident in SHR.

Male SHR showed larger myelin sheaths than females, achieving statistical significance in both segments on the right side, while the $\mathrm{G}$ ratio was generally smaller in males, with significant differences in both distal segments. For WKY, myelin sheath area and $G$ ratio were similar for both genders.

Myelinated fiber diameter ranged from 1 to $13 \mu \mathrm{m}$ in SHR, displaying bimodal distribution with peaks of frequency at 2.5-3 and 7.5-8.5 $\mu \mathrm{m}$. Myelinated axon diameter ranged from 0.5 to $8.5 \mu \mathrm{m}$, displaying unimodal distribution with a peak of frequency at $4.0 \mu \mathrm{m}$. Although the histograms for female SHR were generally skewed to the left, no differences were observed between genders for either distribution. In WKY animals, the distribution of myelinated fiber diameter was also bimodal, with peaks at 2.5-3 and 7.5-8.0 $\mu \mathrm{m}$ and range from 1.5 to $13 \mu \mathrm{m}$. Myelinated axon distribution was also bimodal, following the shape of the myelinated fiber histograms. Regarding SHR nerves, no differences were observed between segments, sides or genders.

The $\mathrm{G}$ ratio distributions were unimodal in all segments studied of both SHR and WKY nerves, with peaks generally at 0.5 or 0.6 , with no differences between segments and sides in the same gender, or between genders for either group.

\section{Myelinated fiber morphometry - comparison between SHR and WKY}

The myelinated fibers and myelin sheaths of male SHR were generally larger than those of male WKY, while the G ratio showed the opposite trend. Comparison of SHR and WKY females showed a trend towards larger fiber and axon sizes (area and diameter) in SHR but only few scattered significant differences were observed. The average $\mathrm{G}$ ratio was generally larger in female WKY, but no significant differences were observed. These findings agree with the differences observed between SHR and WKY in the fiber size and $\mathrm{G}$ ratio distribution histograms. There was a clear and significant reduction in the frequency of small myelinated fibers in both male and female SHR, with no shift on the histograms. The frequency of large myelinated fibers was similar for the two strains, whereas the $\mathrm{G}$ ratio distribution differed between them. Only about $40 \%$ of the phrenic nerve myelinated fibers of male and female SHR presented $\mathrm{G}$ ratios between 0.6 and 0.7 , as opposed to $60 \%$ in WKY animals. Also, more than $25 \%$ of SHR myelinated fibers presented $\mathrm{G}$ ratios smaller than 0.4 , while this percentage was never above $15 \%$ in WKY animals.

\section{Discussion}

\section{Animal model}

SHR, initially bred in the WKY strain, are widely used to study the natural history, genetic determinants and physiopathological alterations of spontaneous hypertension. They are also used in studies involving abnormal control of breathing (9) and male SHR have been shown to exhibit increased post-sigh apneas during sleep compared to normotensive controls (21). Nevertheless, it is intriguing that information about ventilatory control in different rat strains does not include SHR. Also, morphological descriptions of the diaphragm and phrenic nerves are available for different rat strains but not for SHR. The structure and morphometry of phrenic nerves in male and female SHR was examined in the present study because: first, the SHR strain is an important model analogous to essential hypertension in humans (22); second, there is scant information in the literature related to increased post-sigh apnea during sleep in this strain (21); third, no information is available about gender differences in the phrenic nerve of SHR. WKY rats were investigated as the normotensive controls for SHR.

\section{Physiological data}

Arterial pressure levels were significantly different between SHR genders as also observed in the literature $(14,23)$, showing that male SHR have higher SAP compared to females. As expected, both male and female WKY showed lower blood pressure levels than SHR, with no difference between WKY genders, as also reported elsewhere (23).

The current study also shows that anesthetized male SHR exhibited higher HR than female SHR, while there was no difference between genders for WKY animals. This finding contrasts with studies from other laboratories (24) that showed higher HR for unanesthetized females compared to males for both SHR and WKY. Nevertheless, other reports have shown a similar HR for WKY genders (25), as also observed in the present study. The differences between our findings and the literature might be mainly due to the fact that anesthetized rats were used in the present study.

\section{Symmetry study and gender differences}

The importance of examining the right and left phrenic 
nerves resides in the fact that there are reports of lateral asymmetry in these nerves for male normotensive rats $(20,26)$. This lateral asymmetry was confirmed in the present study for both genders in SHR, with the left phrenic nerve usually being smaller than the right nerve, particularly in the distal segment. Nevertheless, no lateral asymmetry was observed in the estimated number of fibers for either SHR gender. For WKY phrenic nerves, the lateral asymmetry (between sides) and longitudinal asymmetry (between segments on the same side) were less evident and, despite the trend towards a smaller nerve on the left side in both genders, there were few significant differences. Lateral symmetry must be taken into account in experiments in which the contralateral nerve of the same animal is used as the control of certain procedures. For the phrenic nerves of SHR and WKY animals (present study) or of Wistar rats $(17,26)$, the use of the contralateral nerve as the control will introduce a bias in any morphological approach.

The exam of proximal and distal segments of a long nerve is important to determine degenerative changes in peripheral nerves, which are known to occur first in the distal portion of the fibers $(27,28)$. A longitudinal (proximal to distal) study of the phrenic nerve was also not reported previously for SHR or WKY. The data obtained in the current study showed a longitudinal asymmetry, especially in the left phrenic nerve for both genders in SHR and WKY, not only for the fascicle size, but mainly for the myelinated fiber area and diameter, with no differences in the axons.

Possible explanations of lateral asymmetry could be: 1) an asymmetrical innervation of the diaphragm, 2) differences in the mechanoreceptor afferents from the pericardium, hepatic parenchyma, hepatic veins, and inferior vena cava in each phrenic nerve, or 3) differences in the post-ganglionic visceral efferents (29). There are suggestions that the right phrenic nerve innervates a larger diaphragm area compared to the left one $(30,31)$, a fact that might result in more myelinated and unmyelinated fibers, including efferents and afferents on the right side nerve. Kostreva and Pontus $(32,33)$ described that afferents from the hepatic veins, liver and inferior vena cava are present on the right phrenic nerve of dogs, while afferents from the pericardium travel on both sides. In addition, vasomotor sympathetic efferents serving the inferior vena cava and hepatic veins may run in the right but not in the left phrenic nerve (33). A study on phrenic nerve development in Wistar rats (29) showed that the right phrenic nerve has 53\% more unmyelinated fibers, which would account for a $32 \%$ larger cross-sectional area of the right phrenic nerve. The authors (29) suggested that the observations made on the afferents and efferents in dogs would also be valid for rats. These afferents and efferents are mainly unmyelinated, a fact that may explain the differences in nerve size and the lack of differences in myelinated fiber number. Further studies on the unmyelinated fibers will be useful to help interpret these differences.
The longitudinal asymmetry observed on the left SHR phrenic nerve could be due to a larger innervation of the pericardium or the existence of receptors within the heart and/or other thoracic structures innervated by the left phrenic nerve in the rat (34), which would enter the nerve and travel only on the upper proximal segments. Findings that ipsilateral phrenic nerve stimulation excited more often cells in the C1-C2 spinal cord segments than the stimulation of the contralateral phrenic nerve in rats suggest a larger innervation of the pericardium by the left phrenic nerve (34). This would explain the larger fascicle on the proximal segment of the left phrenic nerve, since the fibers that innervate the pericardium are present only in this segment, leaving the nerve before it enters the diaphragm. The trend for larger fascicular area on the proximal segments of both sides and in both genders, associated with a smaller fiber percentage of occupancy without a larger number of myelinated fibers, is a morphometric suggestion that the proximal segments have a larger number of unmyelinated fibers, which could be the visceral afferents and/or efferents, not investigated in the present study. It has been postulated that nerve fascicles are larger in heavier animals, regardless of their age. The phrenic nerve fascicles were generally larger in male SHR, as were the myelinated fibers, despite the lack of a statistical significance. This observation may suggest that the higher blood pressure levels in male SHR did not affect the normal development and growth of the fascicle and fibers. Although Sabbatini et al. (35) showed an impaired vascular supply for peripheral nerves in hypertension, no significant differences were found in vessel morphology or capillary percentage of occupancy between segments, sides or genders. These findings also indicate that hypertension did not affect the phrenic nerves of 20-week-old SHR.

SHR gender differences were evident regarding fascicle size and significant regarding the number and density of myelinated fibers, while the morphometric parameters of the myelinated fibers and respective axons were mostly similar for males and females. Similar differences between genders were observed in WKY rats.

A larger number of myelinated fibers in the phrenic nerve might suggest that the diaphragm of female rats receives more motor fibers than the diaphragm of male rats. This finding might explain why the respiratory frequency of female rats responds more efficiently than that of male rats under hypoxia (4) or hyperoxia (2), with the diaphragm of female rats having better motor innervation. Gender differences in respiration emerged from passive mechanical properties of the respiratory system, with dynamic compliance being higher and resistance being lower in female rats (4). Nevertheless, a differentiated motor innervation of the diaphragm cannot be ruled out as also being responsible for these differences. The number of fibers in a peripheral nerve is constant throughout life (16). Thus, the number of fibers in a peripheral nerve cannot be correlated with age or gender, being an intrinsic characteristic of each nerve. 


\section{Strain differences}

The most important differences between the phrenic nerves of WKY rats and SHR were a larger nerve size (area and diameter) and a larger number and density of myelinated fibers in WKY rats. On the other hand, SHR myelinated fibers were generally larger on average, especially in males, compared to WKY, as was the average area of the myelin sheath. Average $G$ ratio of the myelinated fibers was generally smaller on SHR, compared to WKY. Likewise, the $G$ ratio histograms for SHR showed a higher frequency of fibers with $\mathrm{G}$ ratios smaller than 0.4 . A correlation between the myelin sheath and the diameter of the respective axon has been known to exist since 1905 (36). Rushton (19) suggested that values between 0.6 and 0.7 would be the $G$ ratio for the maximum conduction velocity of a myelinated fiber. Since most of the phrenic nerve myelinated fibers in WKY have a $G$ ratio of $0.6-0.7$, this result suggests that these fibers may conduct at their maximum speed. In contrast, the phrenic nerve fibers of male and female SHR have $\mathrm{G}$ ratio values compatible with axonal atrophy what could account for a smaller conduction velocity of these fibers.

Myelinated fiber histograms were bimodal and similarly distributed in both SHR genders. A bimodal distribution of the phrenic nerve myelinated fibers was also shown for Wistar rats $(17,37)$ and seems to be the normal pattern of the phrenic nerves in rats. Gottschall (37) has shown that both sensory and motor myelinated fibers of the phrenic nerves have a bimodal distribution, with the sensory myelinated fibers skewed to the left. Thus, the first peak of fibers of small diameters likely corresponds to sensory myelinated fibers, while the second peak of larger fibers corresponds to motor myelinated fibers. Comparison with WKY histograms clearly demonstrated a reduction in the frequency of smaller fibers in the phrenic nerves of SHR. The small myelinated fibers of the phrenic nerves are mostly afferent. It was demonstrated that afferent fibers running in the phrenic nerves connect centrally with respiratory, cardiac acceleratory and vasomotor centers (38). A reduction in the number of these fibers in SHR may lead to an impairment of the modulation of the respiratory motor drive by these fibers $(39,40)$. Our observation of a larger number and density of Schwann cell nuclei in WKY phrenic nerves is in agreement with the larger number of fibers, especially the small ones, with smaller internodes.

Electric stimulation of phrenic nerve afferents has an inhibitory effect on phrenic motoneuron activity (39). Fryman and Frazier (40) have shown that the effect of phrenic nerve afferent feedback from the diaphragm is masked by the influence of vagal inputs and may not play a role in affecting respiratory motor drive during eupneic breathing. However, they may play an important role in shaping the motor response to changes in length-tension characteristics of the diaphragm due to postural changes or added loads to breathing (40). Also, it has been suggested that free nerve endings on the diaphragm might contribute to ventilatory changes during sustained diaphragmatic work (39).

Strain and gender differences in the number of phrenic nerve myelinated fiber is described for the first time in this experimental model of hypertension, indicating the need for thorough functional studies of this nerve in male and female SHR. Further investigations of phrenic nerve function in WKY and SHR will contribute to the understanding of the morphological findings described in this study.

\section{Acknowledgments}

We thank Mr. Antônio Renato Meirelles e Silva, Experimental Neurology Laboratory, and Ms. Maria Tereza Maglia, Electron Microscopy Laboratory, School of Medicine of Ribeirão Preto, USP, for excellent technical support. Research supported by FAPESP (\#04/09139-2, \#06/03200-7, \#09/16748-9, and \#09/50389-6), CNPq (\#303802/2006-5, and \#202079/2007-4) and FAEPA (Fundação de Apoio ao Ensino, Pesquisa e Assistência, Hospital das Clínicas, Faculdade de Medicina de Ribeirão Preto). A.R. Rodrigues was the recipient of a Master's fellowship from CNPq and R.S. Ferreira was the recipient of a Master's fellowship from CAPES while this study was being carried out.

\section{References}

1. Golder FJ, Zabka AG, Bavis RW, Baker-Herman T, Fuller DD, Mitchell GS. Differences in time-dependent hypoxic phrenic responses among inbred rat strains. J Appl Physiol 2005; 98: 838-844.

2. Strohl KP, Thomas AJ, St Jean P, Schlenker EH, Koletsky RJ, Schork NJ. Ventilation and metabolism among rat strains. J Appl Physiol 1997; 82: 317-323.

3. Forster HV, Dwinell MR, Hodges MR, Brozoski D, Hogan GE. Do genes on rat chromosomes 9, 13, 16, 18, and 20 contribute to regulation of breathing? Respir Physiol Neurobiol 2003; 135: 247-261.

4. Mortola JP, Saiki C. Ventilatory response to hypoxia in rats: gender differences. Respir Physiol 1996; 106: 21-34.
5. Comerford T, Fitzgerald MJ. Motor innervation of rodent diaphragm. J Anat 1986; 149: 171-175.

6. Bisschop A, Gayan-Ramirez G, Rollier H, Dekhuijzen PN, Dom R, de Bock V, et al. Effects of nandrolone decanoate on respiratory and peripheral muscles in male and female rats. J Appl Physiol 1997; 82: 1112-1118.

7. Calverley PM. Blood pressure, breathing, and the carotid body. Lancet 1999; 354: 969-970.

8. Leung RS, Bradley TD. Sleep apnea and cardiovascular disease. Am J Respir Crit Care Med 2001; 164: 2147-2165.

9. Schlenker EH, Tamura T, Gerdes AM. Gender-specific effects of thyroid hormones on cardiopulmonary function in SHHF rats. J Appl Physiol 2003; 95: 2292-2298. 
10. McMullan S, Pilowsky PM. The effects of baroreceptor stimulation on central respiratory drive: a review. Respir Physiol Neurobiol 2010; 174: 37-42.

11. Simms AE, Paton JF, Allen AM, Pickering AE. Is augmented central respiratory-sympathetic coupling involved in the generation of hypertension? Respir Physiol Neurobiol 2010; 174: 89-97.

12. do Carmo JM, Huber DA, Castania JA, Fazan VP, Fazan R $\mathrm{Jr}$, Salgado HC. Aortic depressor nerve function examined in diabetic rats by means of two different approaches. $J$ Neurosci Methods 2007; 161: 17-22.

13. Sato KL, do Carmo JM, Fazan VP. Ultrastructural anatomy of the renal nerves in rats. Brain Res 2006; 1119: 94-100.

14. Licursi de Alcantara AC, Salgado HC, Sassoli Fazan V. Morphology and morphometry of the vagus nerve in male and female spontaneously hypertensive rats. Brain Res 2008; 1197: 170-180.

15. Fazan VP, Salgado HC, Barreira AA. Aortic depressor nerve myelinated fibers in acute and chronic experimental diabetes. Am J Hypertens 2006; 19: 153-160.

16. Jeronimo A, Jeronimo CA, Rodrigues Filho OA, Sanada LS, Fazan VP. A morphometric study on the longitudinal and lateral symmetry of the sural nerve in mature and aging female rats. Brain Res 2008; 1222: 51-60.

17. Rodrigues Filho OA, Fazan VP. Streptozotocin induced diabetes as a model of phrenic nerve neuropathy in rats. $J$ Neurosci Methods 2006; 151: 131-138.

18. Auer RN. Automated nerve fibre size and myelin sheath measurement using microcomputer-based digital image analysis: theory, method and results. $J$ Neurosci Methods 1994; 51: 229-238.

19. Rushton WA. A theory of the effects of fibre size in medullated nerve. J Physiol 1951; 115: 101-122.

20. Fazan VP, Rodrigues Filho OA, Jordao CE, Moore KC. UItrastructural morphology and morphometry of phrenic nerve in rats. Anat Rec 2009; 292: 513-517.

21. Carley DW, Trbovic S, Radulovacki M. Sleep apnea in normal and REM sleep-deprived normotensive Wistar-Kyoto and spontaneously hypertensive (SHR) rats. Physiol Behav 1996; 59: 827-831.

22. Amenta F, Di Tullio MA, Tomassoni D. Arterial hypertension and brain damage - evidence from animal models (review). Clin Exp Hypertens 2003; 25: 359-380.

23. VIkovicova J, Javorkova V, Pechanova O, Vrbjar N. Gender difference in functional properties of $\mathrm{Na}, \mathrm{K}$-ATPase in the heart of spontaneously hypertensive rats. Life Sci 2005; 76 : 971-982.

24. Chen Y, Chandler MP, DiCarlo SE. Daily exercise and gender influence postexercise cardiac autonomic responses in hypertensive rats. Am J Physiol 1997; 272: H1412-H1418.

25. Kahonen M, Tolvanen JP, Sallinen K, Wu X, Porsti I. Influence of gender on control of arterial tone in experimental hypertension. Am J Physiol 1998; 275: H15-H22.

26. Fraher JP. Myelin-axon relationships in the rat phrenic nerve: longitudinal variation and lateral asymmetry. J Comp Neurol 1992; 323: 551-557.

27. Ceballos D, Cuadras J, Verdu E, Navarro X. Morphometric and ultrastructural changes with ageing in mouse peripheral nerve. J Anat 1999; 195 (Part 4): 563-576.

28. Lima NT, Fazan VP, Colafemina JF, Barreira AA. Recurrent laryngeal nerve post-natal development in rats. $J$ Neurosci Methods 2007; 165: 18-24.

29. Song A, Tracey DJ, Ashwell KW. Development of the rat phrenic nerve and the terminal distribution of phrenic afferents in the cervical cord. Anat Embryol 1999; 200: 625643.

30. Hebb CO, Krnjevic K, Silver A. Acetylcholine and choline acetyltransferase in the diaphragm of the rat. J Physiol 1964; 171: 504-513.

31. Laskowski MB, Norton AS, Berger PK. Branching patterns of the rat phrenic nerve during development and reinnervation. Exp Neurol 1991; 113: 212-220.

32. Kostreva DR, Pontus SP. Pericardial mechanoreceptors with phrenic afferents. Am J Physiol 1993; 264: H1836-H1846.

33. Kostreva DR, Pontus SP. Hepatic vein, hepatic parenchymal, and inferior vena caval mechanoreceptors with phrenic afferents. Am J Physiol 1993; 265: G15-G20.

34. Razook JC, Chandler MJ, Foreman RD. Phrenic afferent input excites C1-C2 spinal neurons in rats. Pain 1995; 63: 117-125.

35. Sabbatini M, Vega JA, Amenta F. Peripheral nerve vascular changes in spontaneously hypertensive rats. Neurosci Lett 1996; 217: 85-88.

36. Donaldson $\mathrm{HH}$, Hoke GW. On the areas of axis cylinder and medullary sheath as seen in cross sections of the spinal nerves in vertebrates. J Comp Neurol 1905; 15: 1-16.

37. Gottschall J. The diaphragm of the rat and its innervation. Muscle fiber composition; perikarya and axons of efferent and afferent neurons. Anat Embryol 1981; 161: 405-417.

38. Kohrman RM, Nolasco JB, Wiggers CJ. Types of afferent fibers in the phrenic nerve. Am J Physiol 1947; 151: 547553.

39. Jammes Y, Buchler B, Delpierre S, Rasidakis A, Grimaud $C$, Roussos C. Phrenic afferents and their role in inspiratory control. J Appl Physiol 1986; 60: 854-860.

40. Fryman DL, Frazier DT. Diaphragm afferent modulation of phrenic motor drive. J Appl Physiol 1987; 62: 2436-2441. 